\title{
THE BEHAVIOUR OF A SINGLE CATALYST PELLET FOR THE SELECTIVE HYDROGENATION OF ETHYNE IN ETHENE
}

\author{
A. N. R. BOS, E. HOF, W. KUPER and K. R. WESTERTERP ${ }^{\dagger}$ \\ Chemical Reaction Engineering Laboratorics, Department of Chcmical Engineering, University of Twente, \\ PO Box 217, 7500 AE Enschede, The Netherlands
}

(Received 2 December 1991; accepted for publication 11 November 1992)

\begin{abstract}
The steady-state and dynamic behaviour of a single $\mathrm{Pd}-\mathrm{Al}_{2} \mathrm{O}_{3}$ catalyst particle is studied for the selective hydrogenation of ethyne in the presence of ethene, without addition of carbon monoxide. The particle-to-gas heat transfer in the reactor is characterized. During selective hydrogenation, not only the ignition and the extinction phenomena but also oscillatory behaviour is observed. The nature of the single and multipeak oscillations is discussed. With a dynamic model, based on relatively simple kinetic equations and an additional slow mechanism, e.g. the formation of ethylidyne on the catalyst surface, the qualitative features of this system can be described.
\end{abstract}

\section{INTRODUCTION}

Selective hydrogenation is a commonly applied method for the removal of ethyne in ethene streams. On an industrial scale, adiabatic packed-bed reactors are used in cracking plants to reduce the $\mathrm{C}_{2} \mathrm{H}_{2}$ content in the ethene typically from $1 \%$ to $5 \mathrm{ppm}$. Palla. dium on alumina is the most widely employed type of catalyst, because of its relatively good selectivity. The reactions involved are:

$$
\begin{gathered}
\mathrm{C}_{2} \mathrm{H}_{2}+\mathrm{H}_{2} \rightarrow \mathrm{C}_{2} \mathrm{H}_{4}, \Delta H_{298 \mathrm{~K}}=-172 \mathrm{MJ} / \mathrm{kmol} \\
\mathrm{C}_{2} \mathrm{H}_{4}+\mathrm{H}_{2} \rightarrow \mathrm{C}_{2} \mathrm{H}_{6}, \Delta H_{298 \mathrm{~K}}=-137 \mathrm{MJ} / \mathrm{kmol} .
\end{gathered}
$$

Recent studies [see e.g. Margitfalvi et al. (1980, 1981)] have revealed that also the direct hydrogenation of ethyne to ethane can take place:

$$
\mathrm{C}_{2} \mathrm{H}_{2}+2 \mathrm{H}_{2} \rightarrow \mathrm{C}_{2} \mathrm{H}_{6}, \Delta H_{298 \mathrm{~K}}=-309 \mathrm{MJ} / \mathrm{kmol} \text {. }
$$

The hydrogenation reactors are known to exhibit thermal runaway: due to some reason, suddenly the hydrogenation of ethene starts full scale. This is accompanied by an increase of the temperature rise over the reactor, because the reaction has to be carried oul with an excess of hydrogen. We have studied the causes of this runaway. As part of these investigations, we also have done experiments with a single catalyst particle. In preliminary experiments, using a very simple set-up, in which the hydrogenation of ethenein the absence of ethyne - was carried out, we have found some interesting phenomena such as a temperature overshoot. We have reported on these previously; see Westerterp et al. $(1985,1986)$. These phenomena induced us to design and construct a new single-particle reactor set-up in which more accurate

\footnotetext{
Author to whom correspondence should be addressed.
}

experiments can be performed, at elevated pressures and using both $\mathrm{C}_{2} \mathrm{H}_{4}$ and $\mathrm{C}_{2} \mathrm{H}_{2}-\mathrm{C}_{2} \mathrm{H}_{4}$ feed gases.

One of the objectives in this new study is to determine experimentally the multiplicity behaviour of a single catalyst particle in the hydrogenation of ethyne in ethene. In the literature, the theory has been elaborated for particle multiplicity, mostly for simple reaction systems with first- or $n$ th-order kinetics. The number of experimental studies in this field is rather limited. Single-particle experiments can provide useful information: Harold and Luss (1985), for example, have shown that experimentally determined bifurcation diagrams can be applied for elucidating some of the features of the intrinsic kinetic expression.

Unexpectedly, in carrying out multiplicity experiments, we have found that in a certain range of operating conditions the catalyst temperature started to oscillate during the hydrogenation of ethyne in ethene. In the literature, oscillations in heterogeneously catalyzed reaction systems have been reported mainly for oxidation reactions; moreover most studies involve the oxidation of $\mathrm{CO}$ or $\mathrm{H}_{2}$ [see e.g. Razón and Schmitz (1987), who have presented a review containing 400 references]. To our knowledge, no oscillations have been found for the selective hydrogenation of ethyne.

We will first discuss the experimental results for the multiplicity and the oscillatory behaviour of a single cylindrical catalyst pellet of the egg shell type. Based on the experimental observations, possible explanations will be proposed and subsequently implemented in mathematical models. The behaviour of such models will be compared qualitatively with the experimentally found phenomena.

\section{EXPERIMENTAI.}

The experimental set-up is shown schematically in Fig. 1. The gases hydrogen, nitrogen and ethene, or a mixture of ethyne in ethene, were supplied from 


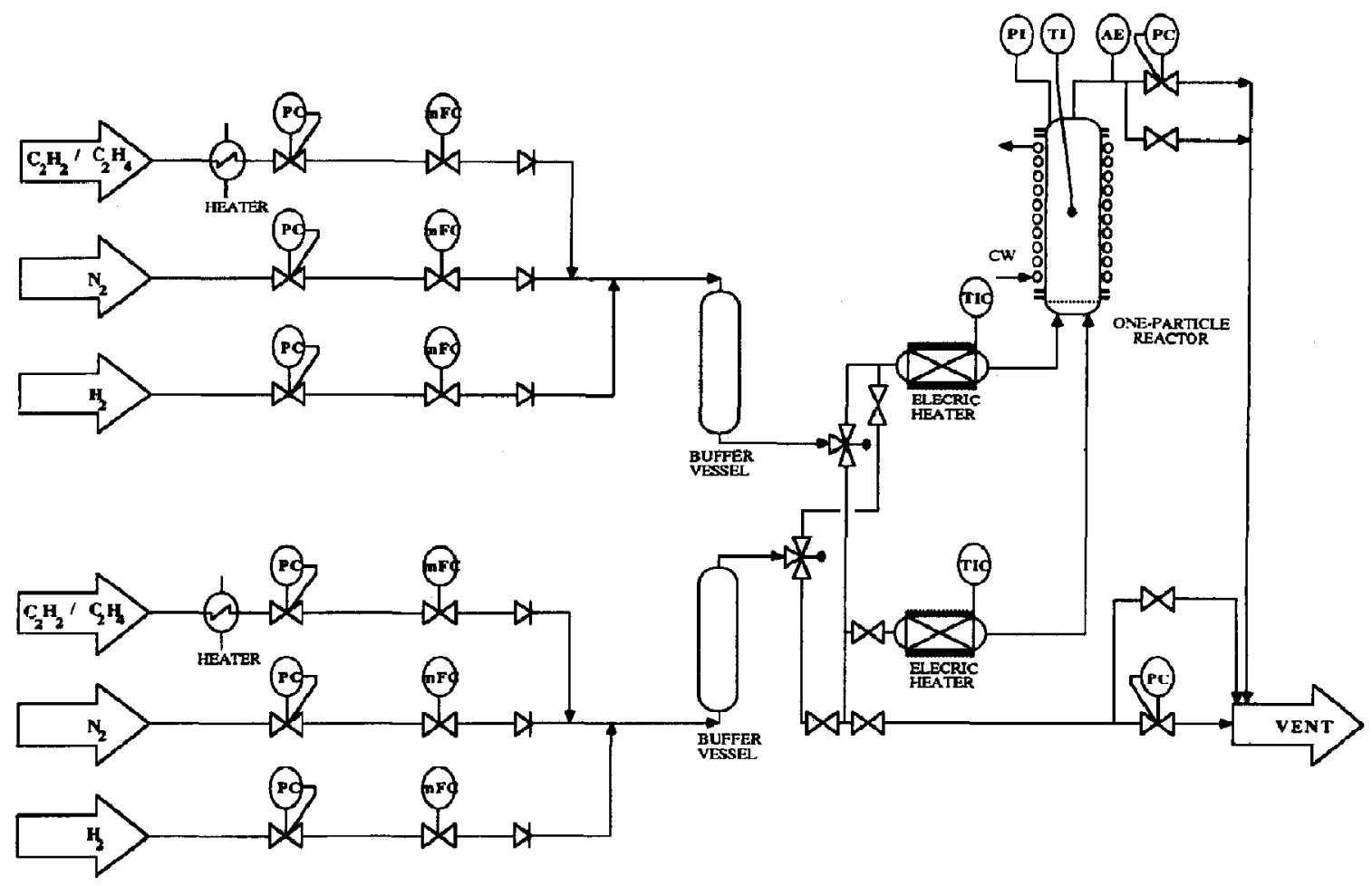

Fig. 1. The experimental set-up. $\mathrm{AE}=$ analysis equipment (gas chromatograph), $\mathrm{CW}=\mathrm{cooling}$ water, $\mathrm{mFC}=$ mass-flow controller, $\mathbf{P C}=$ pressure controller, $\mathbf{P I}=$ pressure indicator, $\mathbf{T I}=$ temperature indicator, TIC = temperature indicator and controller.

bottles. Because of the danger of self-explosion of ethyne, the partial pressure of ethyne must be kept low and, thus, ethyne and ethene could not be mixed on-line at elevated pressures. Mixtures of ethyne and ethene were made by evacuating a bottle, filling it with ethyne until e.g. 0.5 bar, followed by a slow addition of ethene to the final pressure of around 40 bar.

At high flow rates, the expansion of the ethyneethene mixture was accompanied by a strong temperature decrease and, therefore, the gas was preheated to prevent freezing of the pressure reducer and flow controller. All flows were measured and controlled by means of six Brooks 5851TRC and 5850TRC thermal mass flow controllers. Two gas supply sections were used, and via a system with two magnetic valves it was possible to pass either one of the gas mixtures through the single-particle reactor. It was also possible to switch between two electric preheaters. The wallcooled reactor was made of stainless steel and had an inner diameter of $0.04 \mathrm{~m}$ and a length of $0.3 \mathrm{~m}$. The maximum pressure was $2 \mathrm{MPa}$, and the pressure was measured and regulated with back-pressure controllers.

The reactor is shown in more detail in Fig. 2. In the reactor several thermocouples were placed to measure the temperatures of the particle and of the gas near the particle and at the inlet, outlet and the wall of the reactor. One thermocouple was used to keep the

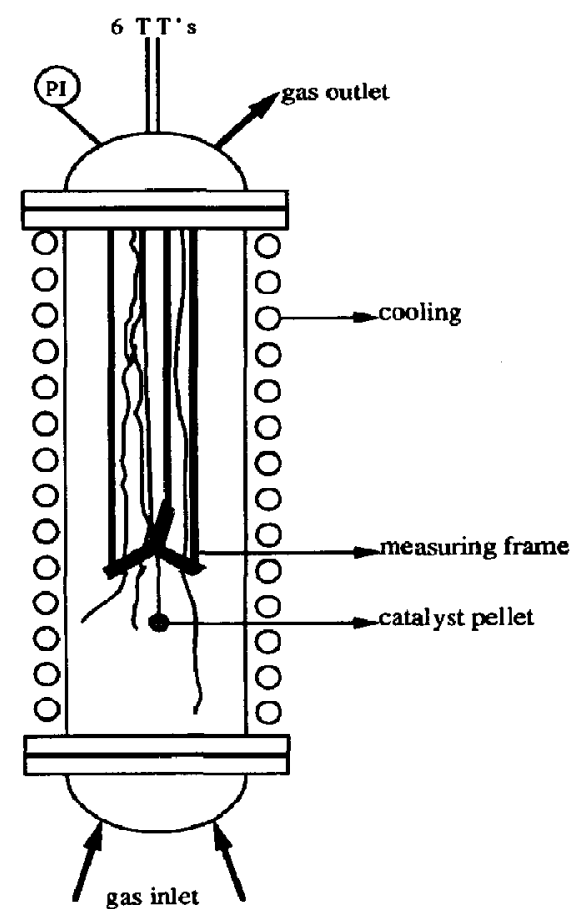

Fig. 2. The single-particle reactor. $\mathbf{P I}=$ pressure indicator, $\mathbf{T T}=$ thermocouple. 
catalyst particle in place as well as to measure the catalyst temperature itself. To this end, after drilling a small hole in the particle, it was mounted on one of the $0.5 \mathrm{~mm}$ thermocouples with the aid of a special cement to ensure good contact. All thermocouples were positioned by means of an internal frame made out of Arlon 1000, a polyetherether ketone, which has a low thermal conductivity. The thermocouples were connected to a Hewlett-Packard Data Acquisition and Control Unit (DACU), whose output was sent to a HP 9816 computer. The data collected were stored and/or presented in graphs.

In most experiments, the reactor pressure, inlet temperature and flow of $\mathrm{N}_{2}$ and $\mathrm{C}_{2} \mathrm{H}_{4}$ or $\mathrm{C}_{2} \mathrm{H}_{2}-\mathrm{C}_{2} \mathrm{H}_{4}$ was kept constant and the amount of $\mathrm{H}_{2}$ varied by changing stepwise. After each change, as soon as the particle temperature varied less than $0.2 \mathrm{~K}$ during $5 \mathrm{~min}$, we assumed that the new steady state had been reached. Some of the properties of the commercial catalyst used in the experiments are listed in Table 1.

\section{EXPERIMENTAL RESULTS}

First, we conducted a number of experiments to determine the particle-to-gas heat transfer. These experiments and their results are discussed in the Appendix.

For the experiments of the hydrogenation of ethene in absence of ethyne, we observed qualitatively the same phenomena as reported previously by Westerterp et al. (1985): after a step change in, for example, the $\mathrm{H}_{2}$ content in the reaction mixture, the catalyst temperature always exhibited an overshoot or an undershoot. These overshoots and undershoots also occurred at elevated pressures. The hydrogen peak [see Westerterp et al.(1986)] could not be produced with this new set-up.

Under certain conditions, the catalyst particle can exhibit more than one steady state, a kinetically controlled lower steady state and an ignited upper steady state where mass transfer is controlling. An example of the experimentally observed ignition, extinction and hysteresis phenomena is shown in Fig. 3. Figure 3 (a) shows these phenomena for the hydrogenation of ethene in the absence of ethyne. Based on the heat transfer experiments discussed in the Appendix, we estimated the heat transfer coefficient to be approximately $40 \mathrm{~W} / \mathrm{m}^{2} \mathrm{~K}$. Figure $3(\mathrm{~b})$ shows a similar experiment but now using a gas mixture of $1.1 \%$ ethyne in nitrogen.

The plots in Fig. 3(a) and (b) can be explained in terms of the basic theories of particle multiplicity

Table 1. Properties of the commercial catalyst

$\mathrm{Pd}-\gamma-\mathrm{Al}_{2} \mathrm{O}_{3}$ cylinders

Diameter $4.4 \mathrm{~mm}$, height $4.4 \mathrm{~mm}$

Catalyst density $1300 \mathrm{~kg} / \mathrm{m}^{3}$

Active metal surface: $350 \mathrm{~m}^{2} / \mathrm{kg}$

Internal surface: $180 \mathrm{~m}^{2} / \mathrm{g}$

Pd content $0.08+0.02 w t \%$

Pd on outer surface only, penetration depth $<0.1 \mathrm{~mm}$

Thermal conductivity $0.5 \mathrm{~W} \mathrm{~m}^{-1} \mathrm{~K}^{-1}$
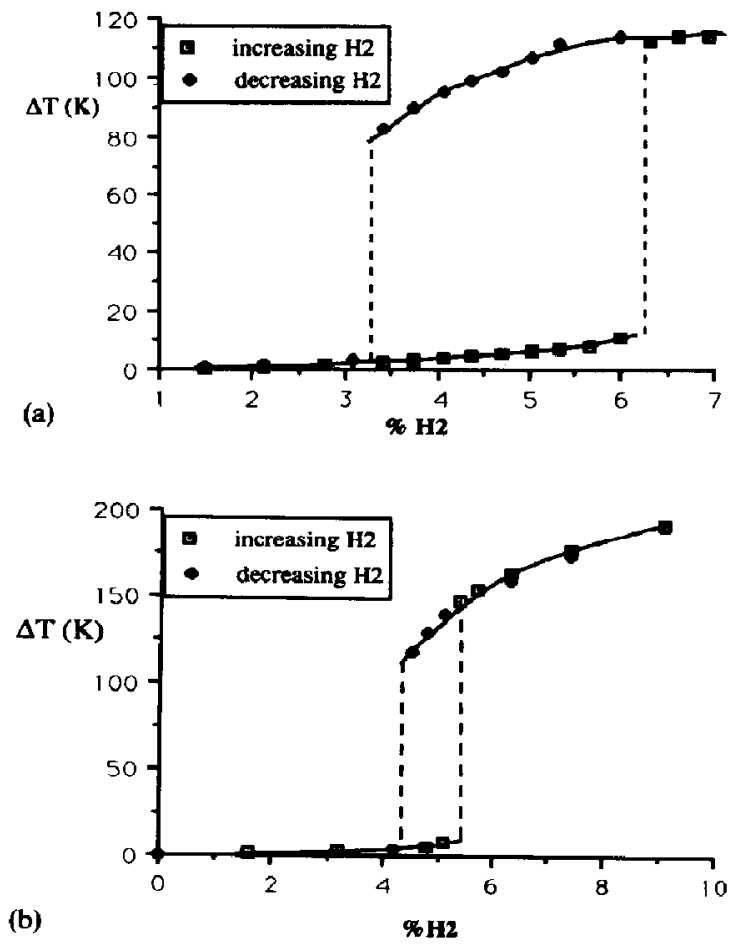

Fig. 3. Ignition and extinction phenomena. Conditions: $P=0.2 \mathrm{MPa}, T_{\mathrm{g}}=308 \mathrm{~K}, v=4 \mathrm{~cm} / \mathrm{s}, R e \approx 30$. (a) $\mathrm{C}_{2} \mathrm{H}_{4}$ hydrngenation, (b) hydrogenation of $1.1 \% \mathrm{C}_{2} \mathrm{H}_{2}$ in $\mathrm{N}_{2}$.

as outlined in many papers and textbooks; see e.g. Westerterp et al. (1987). Here we merely show these experimental results to confirm that particle multiplicity due to external transfer resistances does occur for our system. Further on we will elaborate on this.

The experiments taught us further that the temperature over- and undershoots, as found previously for the hydrogenation of ethene [see Westerterp et al. (1985)] also occur in the presence of $\mathrm{C}_{2} \mathrm{H}_{2}$. Compared to the experiments with only ethene hydrogenation, it generally took much longer-typically $20 \mathrm{~min}-$ before the final steady-state temperature was reached, which is 3 to 4 times longer than in the absence of $\mathrm{C}_{2} \mathrm{H}_{2}$. This points to the presence of a slow mechanism, with a time constant of the order of several minutes.

Unexpectedly - because it has not been reported in the literature-we found during the hydrogenation of ethyne that the catalyst temperature started to oscillate. The oscillations occurred only if ethyne was hydrogenated: no oscillations were found for hydrogenation of ethene in the absence of ethyne. The oscillations started at relatively low $\mathbf{H}_{2}$ contents, below ignition, i.e., at low values of the temperature difference $\Delta T$ between the gas and solid.

This is illustrated in Fig. 4(a), where we show the oscillation occurring for the hydrogenation of ethyne in ethene with $1.3 \%$ of hydrogen, the gas temperature being $308 \mathrm{~K}$, the pressure $0.2 \mathrm{MPa}$ and the gas velocity $0.3 \mathrm{~m} / \mathrm{s}$. The single-peak oscillation had a period 

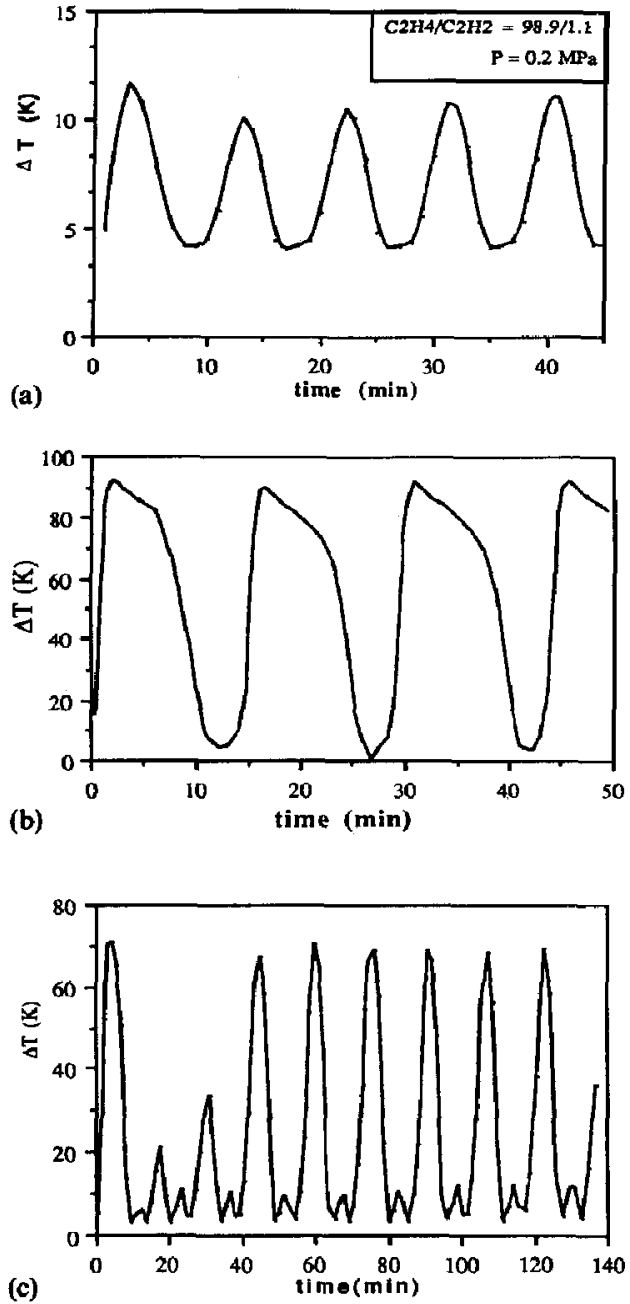

Fig. 4. Single-peak (a) and (b) and multi-peak (c) oscillations observed in the selective hydrogenation. Conditions: $P=0.2$ $\mathrm{MPa}, T_{g}=309 \mathrm{~K}, v \approx 0.3 \mathrm{~m} / \mathrm{s}, R e \approx 240 . \mathrm{H}_{2}$ content: (a) $1.3 \mathrm{vol} \%$, (b) $2.3 \%$, (c) $2.5 \%$.

of approximately $10 \mathrm{~min}$. If the $\mathrm{H}_{2}$ content was increased, also the amplitude of the oscillation increased. At higher $\mathrm{H}_{2}$ contents, we sometimes observed not a single peak but a more complex multipeak oscillation. This is illustrated in Fig. 4(c), presenting the oscillations of the particle temperature at $2.3 \%$ of hydrogen, at further essentially the same conditions as for Fig. 4(a). Figure 4(b) shows a regular single-peak oscillation with a period of around $15 \mathrm{~min}$ accurring at a hydrogen content of $2.5 \%$. At even higher $\mathrm{H}_{2}$ contents, the catalyst temperature ceased to oscillate and always remained in an ignited steady state, with a large difference between the gas and solid temperatures.

The influence of the hydrogen content on the oscillatory behaviour is further illustrated in Fig. 5. This figure shows a series of experiments where the $\mathbf{H}_{2}$ content had been increased in small steps from $1.4 \%$
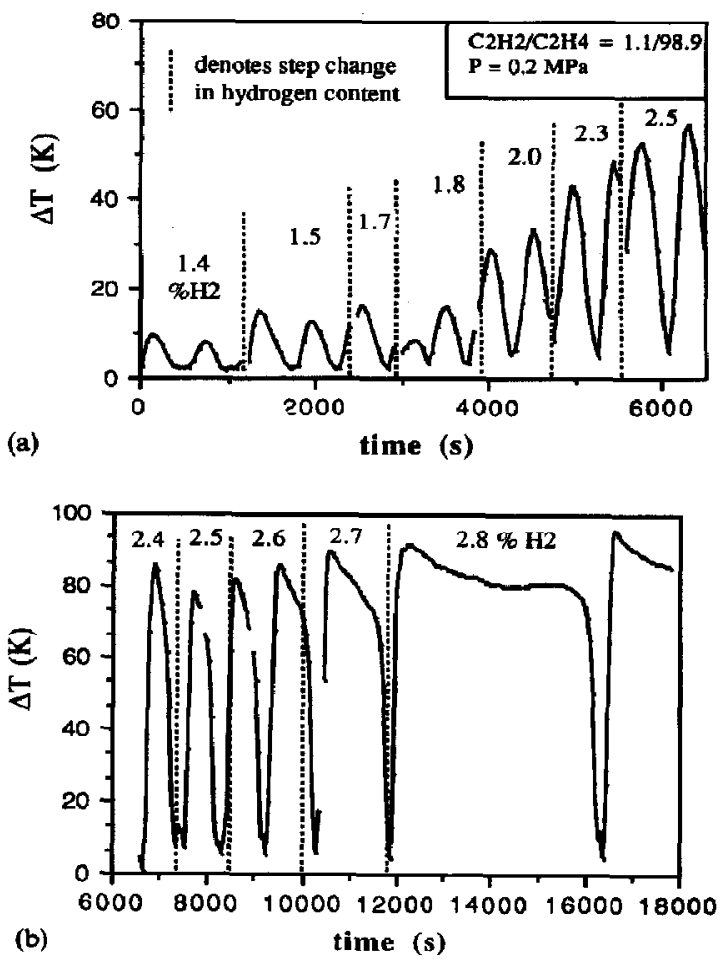

Fig. 5. The influence of the $\mathbf{H}_{2}$ content on the oscillatory behaviour.

to finally $2.8 \%$. These steps are indicated by the dashed vertical lines in Fig. 5. At low hydrogen contents, a single peak oscillation with a low amplitude and a period of approximately $10 \mathrm{~min}$ was found. Up to a hydrogen content of around $2.3 \%$, after every stepwise increase of the hydrogen content, the amplitude of the oscillation $\Delta T_{\max }-\Delta T_{\min }$ increased, while the period remained of the order of $10 \mathrm{~min}$. However, as can be seen in more detail in Fig. 5(b), between 2.3 and $2.6 \% \mathrm{H}_{2}$, the system seemed to bifurcate to a different type of oscillation: the amplitude now remained more or less constant, while the period increased strongly. The cooling of the particle became slower and sometimes a small second peak appeared. Note that at $2.8 \% \mathrm{H}_{2}$ the period of the main oscillation had increased to $70 \mathrm{~min}$ and a sort of plateau appeared. This plateau could easily lead to the erroneous conclusion that a steady state had been reached. At a further increase of the hydrogen content above $2.8 \%$, the oscillations disappeared.

\section{MODEL FORMULATION}

For the time being, we can only speculate about the causes of the oscillations observed. As can be shown rigorously with e.g. a Hopf bifurcation analysis, or felt intuitively considering the period of the oscillations, which is much longer than the thermal relaxation time of the pellet, the oscillations cannot be induced by an interaction of reaction with heat and mass transfer. 
This is possible only for very small particles or thin wires; see e.g. Luss (1975). It must also be realized that the catalyst is of the egg shell type, so that the theoretically possible wealth of complex phenomena due to internal gradients is not relevant in our case. We believe that the oscillations are kinetically induced by means of an interaction between the main reactions and an additional slow mechanism.

Below we will discuss the formation of ethylidyne $\left(\mathrm{C}-\mathrm{CH}_{3}\right)$ as a possible physico-chemical explanation for the presence of an additional slow mechanism. Other factors might be the capacity of Pd to dissolve large amounts of $\mathrm{H}_{2}$ and the formation of two different Pd hydride phases, with different activities; see Bos and Westerterp (1992).

Figure 6 shows a simplified reaction scheme for the selective hydrogenation of mixtures of ethyne in ethene over a Pd catalyst. Besides the two main reactions, also a direct route from ethyne to ethane, via an adsorbed species denoted as $\mathrm{X}$, can exist. It has been shown by spectroscopic techniques [see e.g. Sassen (1989) and Margitfalvi et al. (1981)] that a number of surface species can be formed on Pd, although not all of them will play an active role during selective hydrogenation. Two of the species that have unequivocally been shown to exist are ethylidyne and ethylidene; see Fig. 7. Ethylidyne can be formed both from adsorbed ethyne and ethene and is relatively stable. Several authors [see e.g. Margitfalvi et al. (1981) and Leviness et al. (1984)] have suggested that these species might play an important role in the direct hydrogenation of ethyne to ethane, rather than in the main hydrogenation reactions of eqs (1) and (2).

If formed, ethylidyne may effectively block the active sites available for the hydrogenation of ethyne to ethene. This blocking is reversible if this species can be removed from the surface, e.g. by further hydrogenation to form ethane. Of course, the exact nature

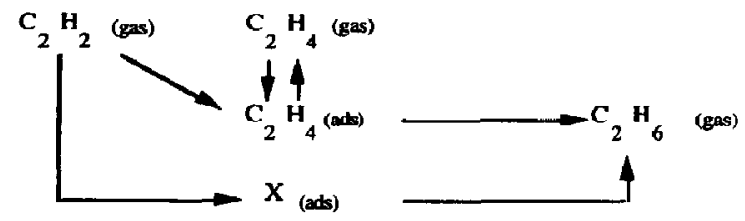

Fig. 6. Simplified reaction scheme for the selective hydrogenation.

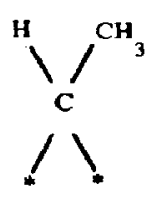

ethylidene

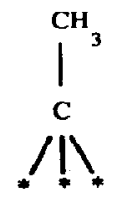

ethylidyne
Fig. 7. Two surface species during selective hydrogenation of ethyne on Pd. and mechanism of the formation of such a surface species is rather complex and still largely unknown. For more information the reader is referred to Bos and Westerterp (1992) and the references therein. Here we merely use the formation of e.g. ethylidyne to explain the presence of a slow reversible blocking and reactivation of the catalyst and the effect on the main reactions (1) and (2). Wicke et al. (1980) have used a similar concept of blocking and reactivation to explain the oscillatory behaviour found for the oxidation of hydrogen.

\section{First-order reaction with reversible blocking}

In order to study the effect of a reversible blocking-reactivation as outlined above, it is helpful to first simplify the reaction system by assuming that only a single first-order reaction occurs. This might be interpreted as being of first order in hydrogen and zeroth order in hydrocarbon; see Bos and Westerterp (1992). Further, we use the same dimensionless temperatures and mass transfer parameter as those used by Wijngaarden and Westerterp (1992) in a steadystate model with a single first-order reaction, and extend their approach to blocking, multiple reactions and particle dynamics.

If the rate of reaction is expressed per unit volume of catalyst, the mass and heat balances are:

$$
\begin{aligned}
& k_{1} C^{i} V_{p}\left(1-\Theta_{B L}\right)=k_{g} A_{p}\left(C-C^{i}\right) \\
& \rho_{p} c_{p} V_{p} \frac{\mathrm{d} T_{p}}{\mathrm{~d} t}=-\alpha A_{p}\left(T_{p}-T_{g}\right) \\
&+k_{1} C^{i} V_{p}\left(-\Delta H_{2}\right)\left(1-\Theta_{B L}\right)
\end{aligned}
$$

where

$$
k_{1}=k_{1}^{0} \exp \left(-\frac{E}{R T_{p}}\right) .
$$

In these equations, $\Theta_{B L}$ denotes the fraction of the active sites that are blocked. First we will examine the steady-state behaviour of this model; thus, the lefthand side of eq. (5) is set to zero. Because we have assumed first-order behaviour, this simple set of nonlinear eqations can be solved analytically. In the absence of blocking, the maximum temperature difference between the gas and the pellet equals $\Delta T_{\text {ad }} L e^{-2 / 3}$; see e.g. Westerterp et al. (1987). It turned out to be convenient to define dimensionless temperature as the temperature divided by this maximum temperature difference:

$$
\begin{gathered}
\theta_{g}=\frac{T_{g}}{\Delta T_{a d}} L e^{2 / 3}, \quad \theta_{p}=\frac{T_{p}}{\Delta T_{a d}} L e^{2 / 3} \\
\theta_{a}=\frac{E}{R \Delta T_{a d}} L e^{2 / 3}
\end{gathered}
$$

where

$$
\begin{aligned}
\Delta T_{a d} & =\frac{C\left(-\Delta H_{1}\right)}{\rho_{g} c_{p g}} \\
L e^{2 / 3} & =\frac{\alpha}{\rho_{g} c_{p g} k_{g}} .
\end{aligned}
$$


The definition of the Lewis number $L e$ follows from the Chilton-Colburn analogy. We further define the parameter $\phi$,

$$
\phi=\frac{k_{g} A_{p}}{k_{1}^{0} V_{p}}
$$

which represents a dimensionless mass transfer coefficient. The steady-state equations (4) and (5) can now be rewritten as

$$
\theta_{p}-\theta_{g}=\frac{1}{1+\phi /\left(1-\Theta_{H L}\right) \exp \left(\theta_{a} / \theta_{p}\right)}
$$

The right-hand side of this equation can be regarded as a dimensionless heat production rate (HPR), scaled between 0 and 1 , and the left-hand side as a dimensionless heat withdrawal rate (HWR). If these are plotted as a function of the pellet temperature, we obtain an S-shaped curve and a straight line for the HPR and HWR, respectively. For the case without blocking, as known, there can be either one, two or three operating points. A discussion of the basic features of multiplicity and associated phenomena can be found in many papers and textbooks, e.g. Westerterp et al. (1987). This will not be repeated here; we focus on the effect of the blocking mechanism.

In Fig. 8 we show the HPR-HWR diagram for a fixed set of parameters and for different values of the blocking fraction $\Theta_{B L}$. There exists a range of values of $\Theta_{B L}$ for which multiplicity occurs: this region is bounded by two critical values, the ignition value and the extinction value. This is also illustrated in Fig. 9, which shows a bifurcation diagram in the $\theta_{p}-\Theta_{B L}$ plane.

It is noteworthy that the effect of $\Theta_{B L}$ is very similar to the effect of the mass transfer parameter $\phi$ : the ratio $\phi /\left(1-\Theta_{B L}\right)$ determines the shape of the HPR curve. Thus, the bifurcation behaviour of the steadystate model with blocking is quite similar to that of the basic model without blocking.

To study the dynamics of the simplified system, we use the time constant of the pellet for cooling or

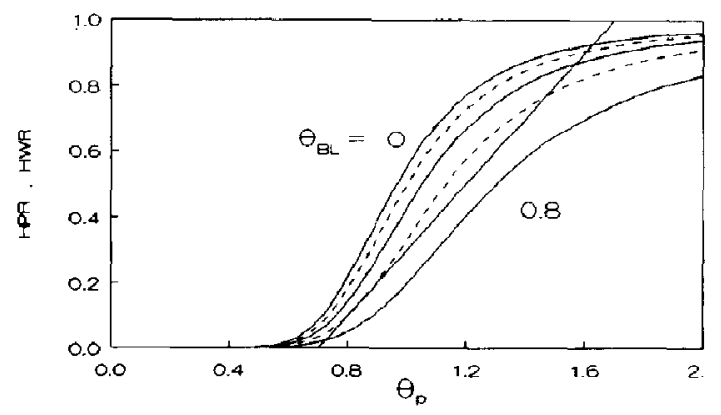

Fig. 8. HPR-HWR diagram for different values of the blocking fraction $\Theta_{B L} . \Theta_{B L}=0,0.2,0.4,0.6$ and $0.8 ; \theta_{g}=0.7$, $\phi=2 \times 10^{-3}, \theta_{\mathrm{a}}=6.0$.

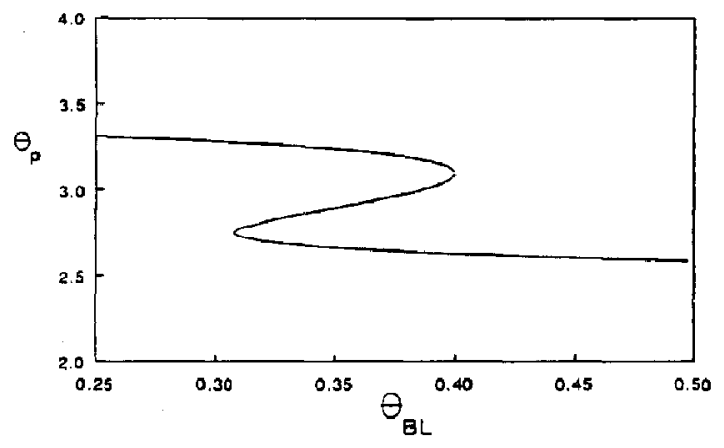

Fig. 9. Bifurcation diagram in the $\theta_{p}-\Theta_{B L}$ plane. $\theta_{\theta}=2.5, \theta=10^{-6}, \theta_{a}=40$.

heating to define a dimensionless time:

$$
\tau=\frac{t}{\tau_{\text {phys }}}, \text { with } \tau_{\text {phys }}=\frac{\left(\rho c_{p} V\right)_{p}}{\alpha A_{p}} .
$$

For our catalyst pellet and experimental conditions, $\tau_{\text {phys }}$ is of the order of $10 \mathrm{~s}$. After substitution of the mass balance equation [eq. (4)], the heat balance of eq. (5) can be written in a dimensionless form:

$$
\frac{\mathrm{d} \theta_{p}}{\mathrm{~d} \tau}=\theta_{g}-\theta_{p}+\frac{1}{1+\phi /\left(1-\Theta_{B L}\right) \exp \left(\theta_{a} / \theta_{p}\right)} .
$$

Different types of blocking-reactivation rate equations are possible. Here we assume that

$$
\frac{\mathrm{d} \Theta_{B L}}{\mathrm{~d} t}=R_{B L}-R_{R E}=k_{B L}\left(1-\Theta_{B L}\right)-k_{R E} \Theta_{B L}
$$

where $k_{B L}$ and $k_{R E}$ are assumed to be temperaturedependent according to

$$
\begin{aligned}
& k_{B L}=k_{B L}^{0} \exp \left(-E_{B L} / R T\right) \\
& k_{R E}=k_{R E}^{0} \exp \left(-E_{R E} / R T\right) .
\end{aligned}
$$

Alternatively, one might also introduce a concentration dependence.

Equation (14) is rendered dimensionless by defining

$$
\begin{aligned}
\tau_{B L} & =\frac{1 / k_{B L}^{0}}{\tau_{\text {phys }}}, \quad m=\frac{k_{R E}^{0}}{k_{B L}^{0}} \\
\kappa & =\frac{E_{R E}}{E_{B L}}, \quad \theta_{B L}=\frac{E_{B L}}{R \Delta T_{a d}} L e^{2 / 3}
\end{aligned}
$$

which then yields

$$
\begin{aligned}
\frac{d \Theta_{B L}}{d \tau}= & 1 / \tau_{B L}\left[\left(1-\Theta_{B L}\right) \exp \left(-\Theta_{B L} / \theta_{p}\right)\right. \\
& \left.-m \Theta_{B L} \exp \left(-\kappa \dot{\theta}_{B L} / \theta_{p}\right)\right] .
\end{aligned}
$$

So, the dynamic model consists of two coupled nonlinear ordinary differential equations, eqs (13) and (17). For appropriate initial conditions, e.g. $\theta_{p}=\theta_{\theta}$ and $\Theta_{B L}=0$ for $\tau=0$, the equations can be solved numerically. We used the Runge-Kutta procedure with adaptive stepsize control given by Press et al. (1988). 
Equation (17) shows that the parameter $\tau_{B L}$ is a measure of the relative "slowness" of the blocking mechanism: at high values of $\tau_{B L}$ the blocking mechanism is slow; at very low $\tau_{B L}$ it will approach an equilibrium value almost instantaneously; when $\tau_{B L}$ goes to infinity, blocking does not occur.

For certain range of parameters, eqs (13) and (17) can exhibit oscillatory behaviour, in contrast to the model without blocking. Considering the large number of parameters, it is practically impossible-and not the purpose of this paper-to map fully the model behaviour. To find parameter values for which the model exhibits oscillatory behaviour, a systematic approach is needed. To this end, we apply a stability analysis for the region nearby a statically steady state, i.e. around the upper or lower steady state; the intermediate steady-state solution is always statically unstable, prohibiting oscillations. If we linearize eqs (13) and (17) around the steady state, i.e. assuming small disturbances $\Delta \theta_{p}$ and $\Delta \Theta_{B L}$ of the steady-state values, we obtain

$$
\begin{aligned}
& \frac{\mathrm{d} \Delta \theta_{p}}{\mathrm{~d} \tau}=a_{11} \Delta \theta_{p}+a_{12} \Delta \Theta_{B L} \\
& \frac{\mathrm{d} \Delta \Theta_{B L}}{\mathrm{~d} \tau}=a_{21} \Delta \theta_{p}+a_{22} \Delta \Theta_{B L}
\end{aligned}
$$

where $a_{i, j}$ are the partial derivatives of the right-hand sides of eqs (13) and (17) evaluated at the steady-state value:

$$
\begin{aligned}
a_{1,1}= & \frac{\theta_{a}}{\theta_{p, s s}^{2}} \frac{\phi}{1-\Theta_{B L, s s}}\left(\theta_{p, s s}-\theta_{g}\right)^{2}-1 \\
a_{1,2}= & \frac{-\phi /\left(1-\Theta_{B L, s s}\right)^{2}}{\left(\theta_{p, s s}-\theta_{g}\right)^{2}} \exp \left(\theta_{a} / \theta_{p, s s}\right) \\
a_{2,1}= & \frac{1}{\tau_{B L}}\left[\left(1-\Theta_{B L, s s}\right) \exp \left(-\theta_{B L} / \theta_{p, s s}\right)\right. \\
- & \left.\kappa m \Theta_{B L, s s} \exp \left(-\kappa \theta_{B L} / \theta_{p, s s}\right)\right] \theta_{B L} / \theta_{p, s s}^{2} \\
a_{2,2}= & \frac{1}{\tau_{B L}}\left[-\exp \left(-\theta_{B L} / \theta_{p, s s}\right)\right. \\
& \left.-m \exp \left(-\theta_{B L} / \theta_{p, s s}\right)\right] .
\end{aligned}
$$

Equations (18) and (19) can be solved analytically, yielding exponential functions of time. The eigenvalues of the linearized system determine the stability in the neighbourhood of the steady-state solution. Oscillation around the steady state, a limit cycle, can only occur if the eigenvalues are complex numbers with positive real parts, which leads to

$$
a_{11}+a_{22}>0 \text { and }\left(a_{22}-a_{11}\right)^{2}+4 a_{12} a_{21}<0 \text {. }
$$

Evaluation of conditions (21) shows that this is the case only if the activation energy $E_{B L}$ of the blocking is higher than that of the reactivation $E_{R E}$.

Conditions (21) can be used to find parameter values for which oscillations around a steady state occur. Yet, we must realize that the above analysis is only a local one and that it loses its validity if we move away from the steady-state solution. Thus, conditions (20) cannot be used to find oscillations where the catalyst pellet oscillates between the upper and the lower steady state, where there are two attractors. In this case, the oscillations can be regarded as a sequence of ignitions and extinctions. A necessary condition for this to occur is that at ignition, i.e. at the lower operating point, the rate of reactivation is higher than the rate of blocking, while at extinction this is reversed. Based on this consideration, a rather complex relation between the parameter values can be derived, which can be used as a guideline to find such oscillations.

Figure 10 shows a few examples of the oscillations obtained with this simple model. The nature of the oscillations can be understood by considering the HPR-HWR diagram shown in Fig. 8. Dynamically, the blocking and reactivation process causes a shift of the HPR curve to the right and to the left, respectively. For example, if we start in the neighbourhood of the lower operating point, relatively small shifts back and forth of the HPR curve lead to a limit cycle around the lower steady state. This shift to the left, due to reactivation, may be so large that the lower operating point no longer exists and subsequently an ignition occurs. At the higher temperature, the blocking mechanism prevails, causing the HPR curve to move back slowly to the right, where eventually an extinction will occur, and the whole process is repeated. Thus, with the simple model described above, it is possible to predict oscillatory behaviour. No multipeak oscillations have been found using this model.

Selective hydrogenation of ethyne, assuming LangmuirHinshelwood kinetics

Although it reflects some of the main features of the oscillatory behaviour, the model outlined and discussed above is, of course, a crude simplification when it is applied to the selective hydrogenation of ethyne in ethene. Here at least two reactions occur and, further, the assumption of simple first-order behaviour in hydrogen and zeroth order in ethyne seems

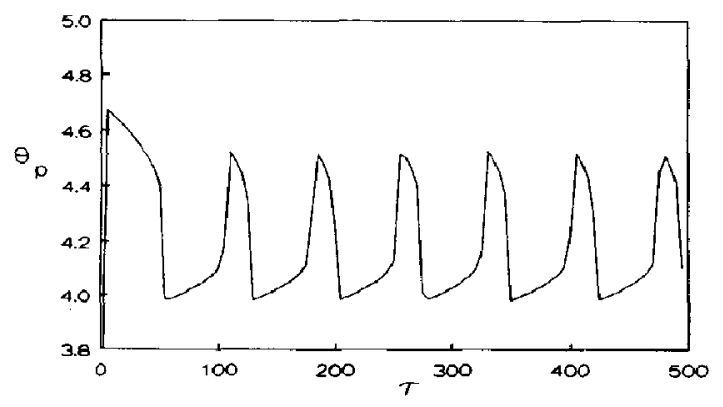

Fig. 10. Oscillatory behaviour calculated with the first-order model. (a) $\theta_{g}=3.8, \phi=5 \times 10^{-9}, \theta_{a}=80, \Theta_{B L}=40$, $\kappa=0.1, \quad m=3 \times 10^{-4}, \tau_{z L}=0.1 ;$ (b) $\theta_{g}=3.8, \phi=5 e^{-9}$, $\theta_{a}=80, \Theta_{a L}=50, \kappa=-4.9, m=7 \times 10^{-30}, \tau_{B L}=0.1$. 
to be appropriate only in a limited range of conditions and, particularly, is questionable near the upper steady state.

The model can easily be extended to a two firstorder reactions system. In that case the bifurcation behaviour is more complex and up to five steady-state solutions are possible; see e.g. Harold and Luss (1985), who studied the bifurcation behaviour of a two firstorder parallel reactions system, without blocking. Over a wide range of parameter values the behaviour of a system of two first-order reactions is very similar to that of a single first-order reaction.

The model becomes significantly more complex if, instead of first-order behaviour, Langmuir-Hinshelwood (LH) kinetics is assumed for reactions (1) and (2). In that case the interface concentration(s) can no longer be evaluated explicitly from the mass balance equation, but must be solved from a set of non-linear equations:

$$
\begin{aligned}
V_{p}\left(1-\Theta_{B L}\right) R_{1}\left(C_{A}^{i}, C_{B}^{i}, C_{C}^{i}\right)= & A_{p} k_{g A}\left(C_{A}-C_{A}^{i}\right) \\
V_{p}\left(1-\Theta_{B L}\right) R_{1}\left(C_{A}^{i}, C_{B}^{i}, C_{C}^{i}\right)+ & R_{2}\left(C_{A}^{i}, C_{B}^{i}, C_{C}^{i}\right) \\
& =A_{p} k_{g B}\left(C_{B}-C_{B}^{i}\right) \\
V_{p}\left(1-\Theta_{B L}\right) R_{2}\left(C_{A}^{i}, C_{B}^{i}, C_{C}^{i}\right)- & R_{1}\left(C_{A}^{i}, C_{B}^{i}, C_{C}^{i}\right) \\
& =A_{p} k_{B C}\left(C_{C}-C_{C}^{i}\right)
\end{aligned}
$$

in which the subscript $A$ denotes ethyne, $B$ hydrogen and $C$ ethene. Thus, instead of only one non-linear equation, the steady-state model for LH kinetics consists of a coupled set of non-linear equations. Qualitatively, the model with LH kinetics can exhibit behaviour not found for simple first-order reactions, for example, isothermal multiplicity and oscillations, which are caused by the interaction between the reaction and the gas to particle mass transfer; see e.g. Eigenberger (1978). This can occur when the effective order in a component changes from positive to negative at increasing concentration of this component, a characteristic of many $\mathrm{LH}$ kinetic expressions. In this case the $H P R$ can have more than one value, wich gives rise to very complex behaviour. We can avoid this by choosing LH kinetic expressions that do not exhibit negative-order behaviour. This agrees with the results of kinetic studies of the selective hydrogenation of ethyne; see Bos and Westerterp (1992) and Bos et al. (1992).

Here we will use the kinetic expression given by Men'shchikov et al. (1975):

$$
\begin{aligned}
& R_{1}=\frac{k_{1} K_{2} K_{H} P_{\mathrm{C}_{2} \mathrm{H}_{2} P_{\mathrm{H}_{2}}}}{\left(1+K_{2} P_{\mathrm{C}_{2} \mathrm{H}_{2}}\right)\left(1+K_{H} P_{\mathrm{H}_{2}}\right)} \\
& R_{2}=\frac{k_{2} K_{4} K_{H} P_{\mathrm{C}_{2} \mathrm{H}_{4} P_{\mathrm{H}_{2}}}}{\left(1+K_{4} P_{\mathrm{C}_{2} \mathrm{H}_{4}}\right)\left(1+K_{H} P_{\mathrm{H}_{2}}\right)} .
\end{aligned}
$$

The model as described by eqs (22)-(24) can be simplified by assuming that the interface concentration of ethene equals the ethene concentration in the gas stream. This is possible if ethene is present in large excess. Defining dimensionless interface concentrations $x_{A}^{i}$ and $x_{B}^{i}$ and the ratio of reaction enthalpies
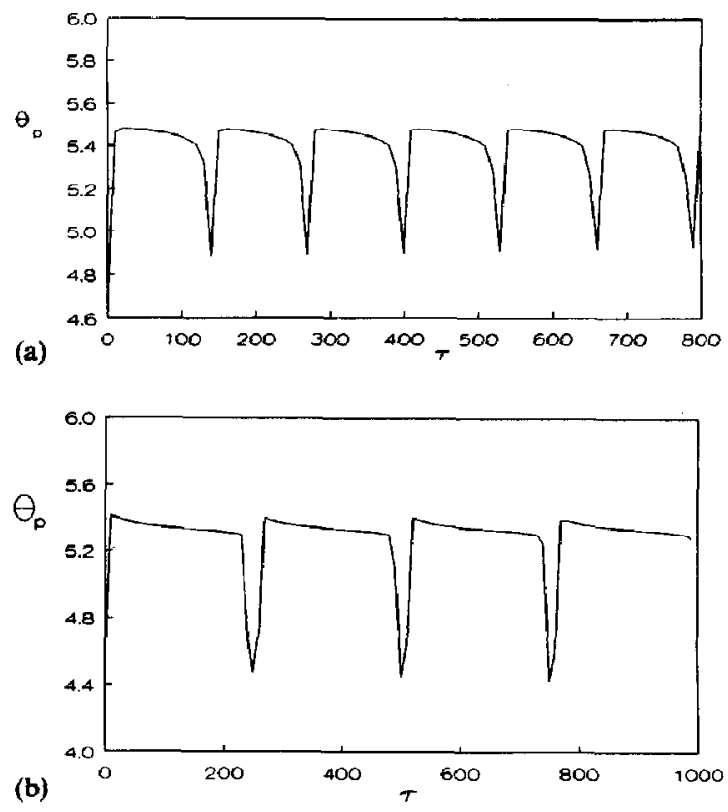

Fig. 11. Oscillatory behaviour calculated with the Langmuir-Hinshelwood model: (a) $\theta_{s}=4.5, \phi=10^{-5}$, $\Theta_{B L}=-10, \quad \kappa=16, \quad m=12 \times 10^{-13}, \quad \tau_{B L}=1000 ; \quad$ (b) $\theta_{a}=4.5, \phi=10^{-5}, \Theta_{B L}=200, \kappa=0.13, m=16 \times 10^{-15}$, $\tau_{B L}=10^{-5}$.

$\boldsymbol{H}$ as

$$
x_{A}^{i}=\frac{C_{A}^{i}}{C_{A}}, \quad x_{B}^{i}=\frac{C_{B}^{i}}{C_{B}}, \quad H=\frac{\Delta H_{2}}{\Delta H_{1}}
$$

the model equations then become

$$
\frac{\mathrm{d} \theta_{p}}{\mathrm{~d} \tau}=\theta_{g}-\theta_{p}+(1-H)\left(1-x_{A}^{i}\right)+H \frac{C_{B}}{C_{A}}\left(1-x_{B}^{i}\right)
$$

$$
\begin{gathered}
\frac{d \Theta_{B L}}{d \tau}=1 / \tau_{B L}\left[\left(1-\Theta_{B L}\right) \exp \left(-\theta_{B L} / \theta_{p}\right)\right. \\
\left.-m \Theta_{B L} \exp \left(-\kappa \theta_{B L} / \theta_{p}\right)\right]
\end{gathered}
$$

where $x_{A}^{i}$ and $x_{B}^{i}$ must be solved from eqs (22)-(24), (25) and (26).

With this more complex model, we calculated the oscillations shown in Fig. 11. The qualitative agreement with the experimentally observed behaviour is quite satisfactory. We were not able to simulate the multipeak oscillations we sometimes observed experimentally. These multipeak oscillations were found only in a few experiments, which indicates that they can be expected only in a very narrow range of model parameters, which in turn implies that this behaviour is easily missed when carrying out simulations. Also, we would bear in mind the relative simplicity of the model as compared to the complex processes occurring on the catalyst surface.

\section{CONCLUDING REMARKS}

We have found experimentally a new oscillatory behaviour in the selective hydrogenation of ethyne in 
ethene on a single $\mathrm{Pd}-\mathrm{Al}_{2} \mathrm{O}_{3}$ catalyst pellet. In order to explain the observed phenomena, we have suggested a reversible blocking mechanism. For this we proposed the formation of ethylidyne, which has recently been shown to be present on the Pd catalyst surface during the selective hydrogenation of ethyne on Pd catalysts, as a precursor of ethane formed from ethyne; see e.g. Sassen (1989) and Margitfalvi et al. (1981).

The real nature of the blocking mechanism can be clarified only by sophisticated surface science techniques. Therefore, in our modelling we have abstained from making assumptions about the exact nature of the blocking mechanism and kept the model as simple as possible. A simple model with a lower number of parameter groups can show more clearly the main effects, which might be obscured by details in complex models. Complex models, incorporating more and, to some extent, speculative details of the mechanisms of both the reactions and the blocking mechanism, contain a large number of parameters on which little or no quantitative information is available. Even for the simple models, the number of parameters is so large that it impedes a complete analysis. We feel that further sophistication of our model is profitable only if additional and quantitative information about the mechanistic details becomes available; the model indicates that these details affect the behaviour of the catalyst pellet significantly. The oscillations might then be used to verify the mechanism proposed, like our study indicates-but by no means proves - the existence of a reversible blocking mechanism probably by the formation of ethylidyne.

Acknowledgements-The authors thank I. de Beer and P. van Berkel for their experimental work, and $G$. H. Banis, $K$. van Bree, W. Leppink, A. H. Pleiter and $H$. Vunderink for technical assistance.

\section{NOTATION}

$a_{i, j}$

partial derivatives of the right-hand side of eqs (13) and (17) with respect to $\theta_{p}$ and $\Theta_{B L}$ for $i=1,2$ and $j=1,2$, respectively

$A_{p} \quad$ external surface area of the catalyst pellet, $\mathrm{m}^{2}$

Bi Biot number $\left(=\alpha d_{p} / \lambda_{p}\right)$

$C$ concentration, $\mathrm{kmol} \mathrm{m}^{3}$

$C^{i} \quad$ interface concentration, $\mathrm{kmol} \mathrm{m}^{3}$

$c_{p} \quad$ heat capacity, $\mathrm{kJ} /(\mathrm{kg} \mathrm{K})$

$d_{p} \quad$ particle diameter, $\mathrm{m}$

$E$ activation energy, $\mathrm{kJ} / \mathrm{kmol}$

$H$ ratio of reaction enthalpies, dimensionless

$\Delta H \quad$ reaction enthalpy, $\mathrm{kJ} / \mathrm{kmol}$

$k^{0} \quad$ frequency factor, $s^{-1}$

$k_{g} \quad$ mass transfer coefficient, $\mathrm{m} / \mathrm{s}$

$K \quad$ kinetic parameter, dimensionless

Le Lewis number, defined by eq. (9), dimensionless

$m \quad=k_{R E}^{0} / k_{B L}^{\mathrm{o}}$

$N u \quad$ Nusselt number $\left(=\alpha d_{p} / \lambda_{g}\right)$, dimensionless

$\operatorname{Pr} \quad$ Prandtl number $\left(=\eta c_{p q} / \lambda_{q}\right)$, dimensionless

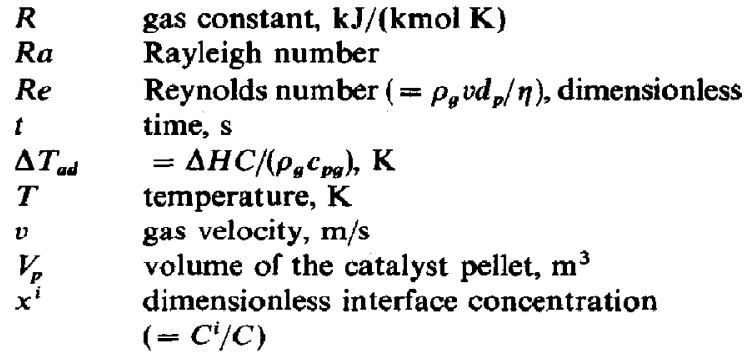

\section{Greek letters}

$\alpha \quad$ heat transfer coefficient, $\mathrm{kW} /\left(\mathrm{m}^{2} \mathrm{~K}\right)$

$\varepsilon \quad$ emission coefficient, dimensionless

$\eta \quad$ dynamic viscosity, $\mathrm{kg} /(\mathrm{m} \mathrm{s})$

$\theta$ dimensionless temperature

$\left(=T / \Delta T_{a d} L e^{2 / 3}\right)$

$\theta_{a}$ dimensionless activation temperature $\left[=(E / R) / \Delta T_{a d} L e^{2 / 3}\right]$

$\Theta_{B L} \quad$ fraction of the surface that is blocked, dimensionless

$\begin{array}{cc}\kappa & =E_{R E} / E_{B L} \\ \lambda & \text { thermal conductivity, } \mathrm{kW}(\mathrm{m} \mathrm{K})\end{array}$

$\rho$ density, $\mathrm{kg} / \mathrm{m}^{3}$

$\sigma \quad$ Stefan-Boltzmann constant, $\mathrm{kW} /\left(\mathrm{m}^{2} \mathrm{~K}^{4}\right)$

$\tau \quad$ dimensionless time $\left(=t / \tau_{\text {phys }}\right)$

$\tau_{B L} \quad=1 /\left(k_{B L}^{0} \tau_{\text {phys }}\right.$, dimensionless

$\tau_{\text {phys }} \quad$ time constant for physical cooling or heating $\left[=\left(\rho c_{p} V\right)_{p} / \alpha A_{p}\right], \mathrm{s}$

$\phi \quad a$ dimensionless mass transfer parameter $\left[=k_{g} A_{p} / k_{1}^{0} V_{p}\right]$

\section{Subscripts}

1,2 reaction (1) or (2)

$A, B, C$ denotes components ethyne, hydrogen and ethene, respectively

$\begin{array}{ll}B L & \text { blocking } \\ g & \text { gas } \\ p & \text { particle } \\ R E & \text { reactivation } \\ s s & \text { steady state } \\ w & \text { reactor wall }\end{array}$

\section{REFERENCES}

Bos, A. N. R. and Westerterp, K. R., 1992, Mechanism and kinetics of the selective hydrogenation of ethyne and ethene. A review. Chem. Engng Process 32(1), 1-22.

Bos, A. N. R., Foeth. F., Bootsma, E., Sleyster, H. and Westerterp, K. R., 1992, A kinetic study of the selective hydrogenation of ethyne and ethene on a commercial $\mathrm{Pd} / \mathrm{Al}_{2} \mathrm{O}_{3}$ catalyst. Chem. Engng Process 32(1), 51-67.

Churchill, S. W., 1977, A comprehensive correlating equation for laminar, assisting, forced and free convection. A.I.Ch.E. J. 23(1), 10-16.

Gnielinski, V., 1975, Berechnung mittlerer Wārme- und Stoffübergangs-koeffizienten an laminar und turbulent überströmten Einzelkörpern mit Hilfe einer einheitlichen Gleichung. Forsch. Ing.-Wes, 41(5), 145-153.

Harold, M. P. and Luss, D., 1985, An experimental study of steady state multiplicity features of two parallel catalytic reactions. Chem. Engng Sci. 40, 39-52.

LeViness, S., Nair, V, Weiss, A. H., Schay, Z. and Guczi, L., 1984, Acetylene hydrogenation selectivity control on $\mathrm{PdCu} / \mathrm{Al}_{2} \mathrm{O}_{3}$ catalysts. J. Molec. Catal. 25, 131-140. 
Luss, D., 1977, Steady state and dynamic behaviour of a single catalytic pellet, in Chemical Reactor Theory-a Review (Edited by L. Lapidus and N. R. Amundson), pp. 191-268. Prentice-Hall, Englewood Cliffs, NJ.

Margitfalvi, J., Guczi, L. and Weiss, A. H, 1980, Reaction routes for hydrogenation of acetylene-ethylene mixtures using a double labelling method. React. Kinet. Catal. Lett. 15, 475-479.

Margitfalvi, J., Guczi, L. and Weiss, A. H., 1981, Reactions of acetylene during hydrogenation on Pd black catalyst. $J$. Catal. 72, 185-198.

Men'shchikov, V. A., Fal'kovich, Yu. G. and Aerov, M. E., 1975, Hydrogenation kinetics of acetylene on a palladium catalyst in the presence of ethylene. Kinet. Catal. 16(2), 1335-1338.

Press, W. H., Flannery, B. P., Teukolsky, S. A. and Vetterling, W. T., 1988, Numerical Recipes. Cambridge University Press, New York.

Ranz, W. E. and Marshall, J. R., 1952, Evaporation of drops. Chem. Engng Prog. 48, 141-146, 173-179.

Razón, L. F, and Schmitz, R. A, 1987, Multiplicities and instabilities in chemically reacting systems - a review. Chem. Engng Sci. 42, 1005-1047.

Sassen, N. R. M., 1989, Ph.D. thesis, Leiden University, The Netherlands.

VDI-Wärme Atlas, 1988, VDI Verlag, Düsseldorf, BRD, 5. Auflage, Fa5-Fa6.

Westerterp, K. R., Kusters, W. C. and Wijngaarden, R. J., 1985, A temperature overshoot on a catalyst pellet. Chem. Engng Sci. 40, 1463-1467.

Westerterp, K. R., Loonen, R. A. and Martens, A., 1986, Rare behaviour of a catalyst pellet; catalyst dynamics. Chem. Engng Sci. 41, 1117-1121.

Westerterp, K. R., van Swaaij, W. P. M. and Beenackers, A. A. C. M., 1987, Chemical Reactor Design and Operation. Wiley, Chichester.
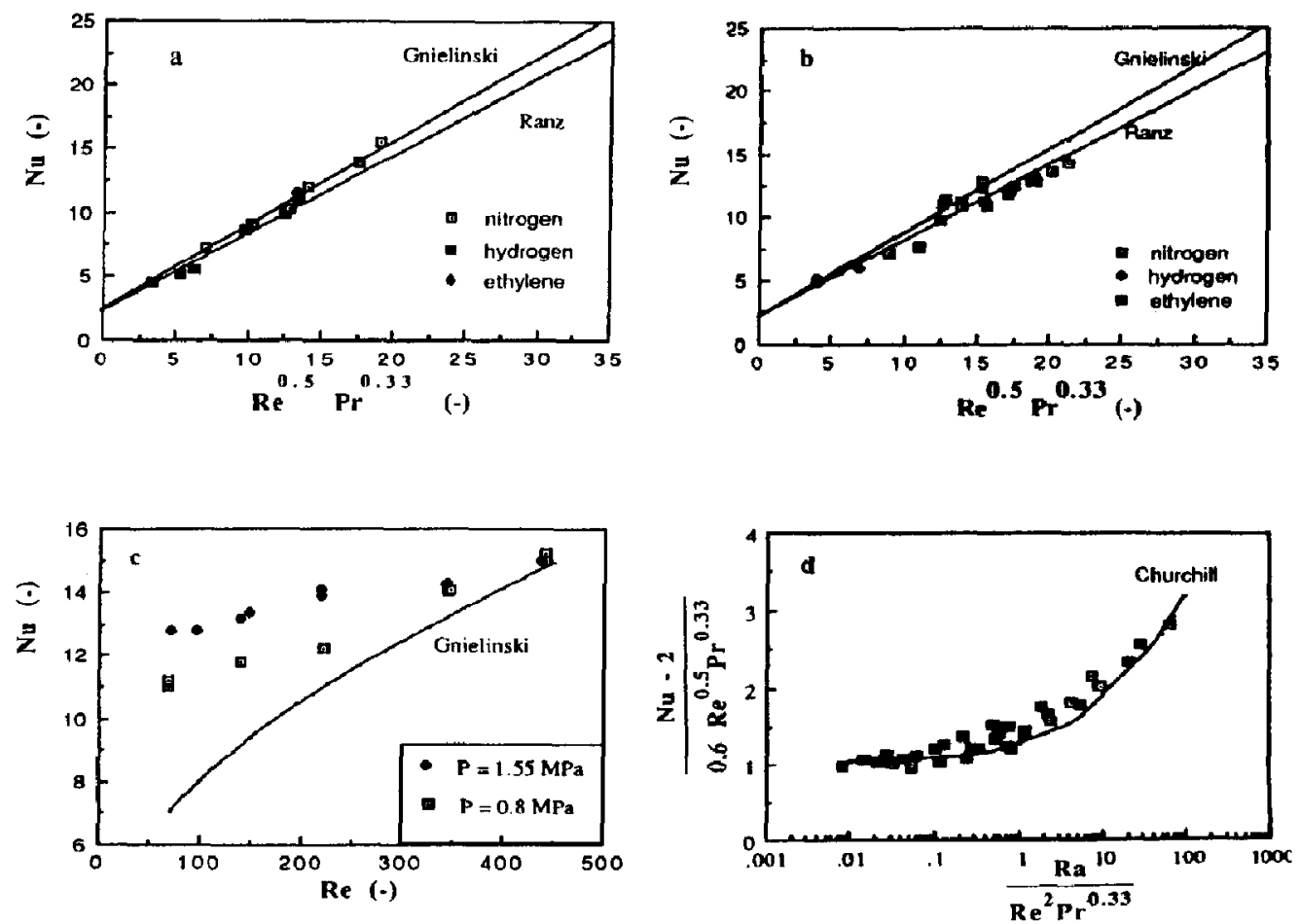

Fig. A1. Nusselt number as a function of the Reynolds number: (a) aluminium sphere, $P<0.3 \mathrm{MPa}$; (b) aluminium cylinder, $P<0.3 \mathrm{MPa}$; (c) aluminium sphere, $P>0.5 \mathrm{MPa}$; (d) alumina sphere, combined free and forced convection; same way of representation as Churchill (1977). 
of $\alpha$ could also be obtained directly without fitting, by rewriting eq. (A1) as

$$
\alpha=\frac{\sigma \varepsilon A_{p} \int_{t_{1}}^{t_{2}}\left[T_{p}^{4}(t)-T_{w}^{4}\right] \mathrm{d} t+\rho_{p} V_{p} \int_{T_{1}}^{T_{2}} c_{p} \mathrm{~d} T}{A_{p} \int_{t_{1}}^{t_{2}}\left[T_{p}(t)-T_{p}(t)\right] \mathrm{d} t}
$$

and using the measured $T_{p}(t)$ and $T_{0}(t)$ profiles for the integrals in eq. (A2). For both methods the values of the first few seconds as well as those at the end of an experiment, where $\left[T_{p}(t)-T_{g}\right]<0.1\left[T_{p}(t=0)-T_{g}\right]$, were omitted. Within the experimental accuracy, both methods yielded the same values.

Equations (A1) and (A2) are valid only if intraparticle gradients can be neglected. A simple criterion for this $B i<0.1$, which can also be written as $N u<0.1 \lambda_{p} / \lambda_{g}$. For the catalyst pellet this criterion is not fulfilled because of its low thermal conductivity and, thus, it was not used to determine the heat transfer coefficient.

\section{Results and discussion}

In Fig. Al the experimentally determined Nusselt numbers are shown as a function of the Reynolds number for the alnminium and non-porous alumina spheres and cylinders. The correlations of Ranz and Marshall (1952) and Gnielinski (1975) are also plotted in these figures. For the characteristic pellet diameter, Gnielinski's recommendation was followed and we took $d_{p}=\left(A_{p} / \pi\right)^{0.5}$. We see from Fig. A1(a) and (b), that for lower pressures of $0.1-0.3 \mathrm{MPa}$ both correlations are adequate for describing the heat transfer in our system.

At higher pressures the experimentally determined Nusselt numbers are higher than those predicted from the Ranz or Gnielinski equation. This is illustrated in Fig. A1(c). The deviation at higher pressures is caused by the increased contribution of free convection. We have described the combined free and forced convection using the method outlined by Churchill and recommended by the VDI-Wärme Atlas (1988); see also Churchill (1977). This is illustrated in Fig. $\Lambda 1(d)$.

From the heat transfer experiments, we conclude that at lower pressures the well-known correlations of Ranz or Gnielinski describe the particle to gas heat transfer adequately, provided a correction for radiation heat loss is taken into account. The importance of the radiative heat transfer depends both on the temperature and on the amount of heat transfer by convection--and, thus, also on the Reynolds number. For our experiments the contribution of radiation to the total heat transfer is of the order 5-20\%. At higher pressures, free convection becomes important and the correlations of Ranz and Gnielinski yield too low values of $\alpha$. Free convection, as shown in Fig. A1(c), is a drawback for the description of the chemical experiments executed under elevated pressures, because the predicton of heat transfer coefficients for combined free and forced convection is more complex and less accurate. 